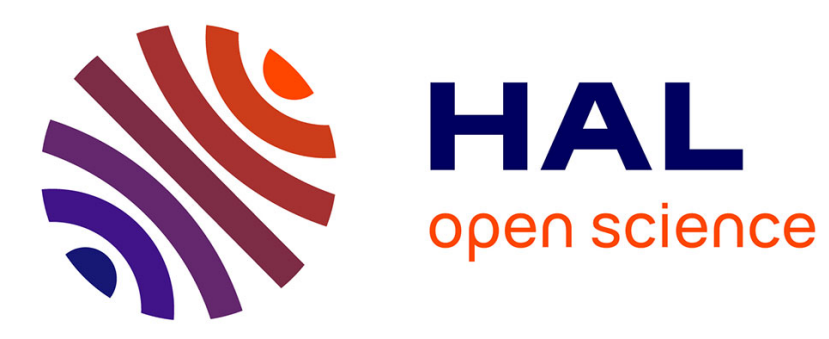

\title{
Diglossie et double langage. Langues et langages d'honneur en Grèce.
}

\author{
Maria Couroucli
}

\section{To cite this version:}

Maria Couroucli. Diglossie et double langage. Langues et langages d'honneur en Grèce.. Langage et Société, 1991, 57, pp.71-92. halshs-00353201

\section{HAL Id: halshs-00353201 https://shs.hal.science/halshs-00353201}

Submitted on 14 Jan 2009

HAL is a multi-disciplinary open access archive for the deposit and dissemination of scientific research documents, whether they are published or not. The documents may come from teaching and research institutions in France or abroad, or from public or private research centers.
L'archive ouverte pluridisciplinaire HAL, est destinée au dépôt et à la diffusion de documents scientifiques de niveau recherche, publiés ou non, émanant des établissements d'enseignement et de recherche français ou étrangers, des laboratoires publics ou privés. 


\section{Maria Couroucli: Diglossie et double langage: langues et langages d'honneur en Grèce, in Langage et Société, 57, 1991 pp. 71-92.}

La langue de la littérature néohellénique ne contient nul message. La langue est le message. La façon de s'exprimer d'un écrivain, c'est l'argument de son livre. (V. Vassilikos, $1989: 211$ ).

En grec moderne, pour dire que quelque chose a été énoncée de manière précise et détaillée, on utilise une expression proche de l'expression française "mettre les points sur les i" ; on dit: "avec le n et le s" (me to ni ke me to sigma). Ces consonnes, qu'on trouve souvent à la fin des mots, relèvent du domaine de l'écrit, de la chose finalisée, du document juridique ou administratif : "en France", ça se dit sti Gallia, mais on écrit is tin Gallian . La diglossie ${ }^{1}$ en Grèce n'est pas un phénomène nouveau : la langue commune byzantine, la koinè , avait déjà évolué indépendament de la langue officielle de l'Etat et de l'Eglise, les puristes ayant même cherché à fixer une version savante de celle-ci comme symbole immuable de la pérénnité de l'Empire chrétien. Cette cassure culturelle de l'hellénisme continue jusqu'au XIXe siècle, lorsque apparaîssent deux langues distinctes, la langue "épurée" (katharevousa) essentiellement écrite, d'une part, et la langue "populaire" (dimotiki v) de l'autre, produit de l'évolution de la langue parlée qui, à son tour, peut se distinguer en plusieurs dialectes régionaux (Patlagean, 1979).

Dans la Grèce moderne, la diglossie reflète l'opposition classique entre le "peuple" et l'"élite". En effet, l'apprentissage de la langue savante, langue de l'écriture par excellence, se fait à l'école : c'est cette langue purifiée des mots "barbares" (elle ne contient pas -en théorie- de mots étrangers), qui prend une forme simplifiée du grec ancien que tout citoyen honorable doit maîtriser. Jusque dans les années 1970, les lois, les textes administratifs, les journaux, les nouvelles à la radio, étaient tous livrés en katharevousa : celle-ci devient l'instrument de la pensée, de la science et des idées nobles. Toute personne qui la maîtrise est réputée faire partie de la classe sociale dominante : à l'opposé du paysan, du petit commerçan, de la ménagère ou du travailleur manuel, les fonctionnaires, les professionnels du droit, les médecins, les prêtres, s'exprimaient en public ou par écrit dans cette langue, l'usage de la langue populaire (dimotiki ) étant réservé à la vie quotidienne, pour exprimer des idées banales et vulgaires. Cette situation change à partir en 1975, lorsque une nouvelle langue mixte qui, selon l'ancienne formule de Mirambel, "limite à la fois le purisme et le vulgarisme" devient langue officielle de l'Etat. La nouvelle koinè est la vraie fille de la diglossie séculaire dans le monde grec : appellée Neoelliniki (koinè néogrecque) elle a été decrite non sans ambiguité comme "une démotique sans extrèmes" (Babiniotis, 1979).

C'est dans le domaine juridique que les choses ont évolué le plus lentement, car la langue savante est réputée plus "complexe" ; notamment plus riche en suffixes, mais surtout possédant des participes avec flexion, elle abonde en phrases secondaires, pour la plus grande joie des rédacteurs de textes juridiques ou administatifs. Ainsi, la langue institutionnelle, la langue de la loi, du pouvoir (mais aussi de l'église), a longtemps résisté à l'introduction de la nouvelle koinè dans les salles des tribunaux. 


\section{DEUX LANGUES, DEUX LANGAGES}

Dans les pages qui suivent, il s'agit d'envisager les effets et les manipulations de la diglossie dans un contexte particulier, là où la langue juridique rencontre et s'oppose à la langue parlée : on examinera deux cas de "crimes d'honneur" tels qu'ils sont décrits dans les procès verbaux des audiences du tribunal de Grande Instance de Iraklio en Crète, dans les années $1970 .^{2}$ Dans cette situation, le dédoublement linguistique s'opère par deux fois.

En premier lieu, il s'agit des deux langues : la "savante" (katharevousa) et la "vulgaire" (dimotiki ). Conformément à l'usage, c'est la première qui domine dans les audiences des tribunaux : les professionnels du droit s'expriment en katharevoussa, et on peut supposer que leur discours est rendu tel quel dans les procès verbaux. Les témoins, par contre, qui sont dans leur grande majorité des paysans, s'expriment en dimotiki . Le scribe du tribunal crétois, tout comme le scribe de Jacques Fournier le faisait pour les dires de villageois de Montaillou, ${ }^{3}$ suit la tradition de sa profession : il transcrit le discours livré en dimotiki dans la langue du tribunal, c'est-à-dire en katharevoussa: "avec les $\mathrm{n}$ et les s". Cela dit, dans le texte du discours des témoins, on y trouve souvent des phrases ou même des passages assez longs, écrits entre guillemets : ce sont justement des "vulgarités" prononcées par les acteurs, des altercations qui contiennent des injures ou des expressions populaires et qui sont rendues dans leur état brut, car sûrement jugées importantes dans le déroulement du débat.

Le deuxième point concerne le langage : tout montre que les acteurs se mettent en situation de "performants" (Herzfeld, 1985), et que chacun joue un rôle bien défini: les locuteurs de la katharevoussa sont ceux qui se réfèrent au code moral de l'EtatNation et donc aux lois de l'Etat, tandis que les locuteurs de la dimotiki sont perçus par les premiers comme des hommes qui, ignorant les institutions nationales, agissent selon le code traditionnel de l'honneur encore en vigueur dans les communautés villageoises. La médiation se fait par les jurés : indigènes mais urbains, proches des uns par le mode de vie et des autres par la tradition, ce sont eux qui tranchent entre les deux langages et qui livrent le verdict.

En d'autres termes, le tribunal est aussi un lieu où les deux langages se télescopent: celui de la loi et la raison d'Etat d'une part et celui de l'idéologie nationaliste de l'autre. En rendant le dit des acteurs et des témoins villageois dans la langue officielle, épurée des "vulgarités" de la langue parlée, c'est comme si, volontairement ou non, on tentait, par le biais du langage national, d'inscrire le cas précis dans un système de valeurs communes, de le "retraduire" (Bourdieu, 1982:41). Ainsi, certains termes du code de l'honneur ou du système de la parenté traditionnel sont remplacés par des mots de la katharevousa : les termes jugés dialectaux, propres à la société locale, n'ont pas leur place dans un tribunal qui, lui, relève de l'Etat national. Dans ce dialogue de sourds un registre se singularise : les termes vernaculaires que les acteurs refusent de prononcer devant les juges sont souvent ceux qui se réfèrent à l'identité masculine, aux valeurs et vertus viriles socialement reconnues dans le système communautaire local. 


\section{LANGAGE DE L'HONNEUR ET IMAGES D'ÉPINAL}

Paradoxalement, le langage national, identitaire, valorise constamment la communauté locale : dans l'imagerie populaire, nourrie par le folklore (même retouchées, les chansons populaires sont toutes en dimotiki dialectale), le vaillant combattant qui libère la patrie des turcs est un berger, un habitant de la montagne, qui parle son dialecte et qui, de manière presque instinctive, se bat pour la Nation. Dans cette même imagerie nationale et populiste, le vaillant montagnard défend le système des valeurs indigène contre celui imposé par le pouvoir central ottoman.

L'opposition Etat central / communauté locale dans la Grèce d'aujourd'hui relève donc d'un paradigme dont les origines se situent dans un système d'Empire, où le pouvoir central était perçu comme "étranger" à la communauté locale, un système où la notion de citoyenneté est absente. ${ }^{4}$

Dans la Crète contemporaine, l'opposition Etat - société traditionnelle semble perdurer et faire partie intégrante du discours indigène (Herzfeld, 1985). La rhétorique paysanne identifie l'Etat à un oppresseur violent qui, comme "L'Ottoman" d'autrefois, agissent de manière arbitraire et repressive vis-à-vis des communautés villageoises. Selon l'analyse indigène, celles-ci, héritières de la culture hellène, agissent selon un code traditionnel qui fait appel à l'honneur consubstentiel, à l'homme dans son identité, et ne peuvent que se confronter aux représentants des autorités, entité lointaine et abstraite. Les habitants des communes de la montagne crétoise, qui a connu la résistance contre tous les envahisseurs, qu'ils soient vénitiens, turcs, ou allemands, ont fini par s'identifier aux images d'épinal dont l'idéologie nationale s'est servie dans sa quête identitaire unificatrice du monde hellène contemporain.

L'opposition recurrente entre, d'une part, la langue et le langage local et, d'autre part, la langue et le langage national reproduit ce mythe d'origine, selon lequel la Grèce se libère de la domination ottomane à l'issue de l'affrontement entre le pouvoir central de l'Empire et les bandes armées nationales : La justice personnelle (dikio ) s'opposerait donc à la justice institutionnelle (dikaiosini), tout comme les droits (dikia) des grecs occupés s'opposaient aux injustices (adika) des autorités turques.

Mettant en cause la légalité et la moralité de tout pouvoir central, les "vrais" crétois ne peuvent que "voler" les biens qui leurs sont interdits par l'arbitraire des serviteurs de l'Etat. Ainsi, par exemple, le vol du bétail en Crète, une forme de compétition masculine répandue parmi les bergers de la Crète occidentale, est érigée en institution traditionnelle par les acteurs eux-mêmes, mais aussi célébrée par les folkloristes originaires de l'île : elle serait une "habitude" prise lors des l'occupation ottomane, lorsque les chrétiens volaient aux infidèles le fruit de leur répression, une habitude devenue coutume (Tsouderos, 1976: 41). D'ailleurs, les fameuses bandes armées de la guerre d'indépendance contre l'Empire ottoman n'étaient-elles pas aussi des bandes de voleurs au sens littéral (Kleftes ), qui prenaient aux riches pour donner aux pauvres? Comme dans le cas des bandits des autres rives de la méditerranée, le syndrome des rebelles primitifs devient une justification des formes de violence censées faire partie de l'organisation communautaire traditionnelle mais réprimées par l'Etat.

Tant pour les acteurs que pour les représentants du pouvoir, l'idéologie indigène qui justifie la violence est une raison supplémentaire pour séparer les deux mondes : les communautés isolées, proches d'un mode de vie d'antan, représentent certes la société traditionnelle dont les valeurs fondent l'idéologie grecque identitaire mais aussi des poches de résistance à la modernité. Une situation qui permet une négotiation 
permanente des limites d'appartenance et qui caractérise la manière dont l'opposition Etat / communauté villageoise a structuré les rapports entre les citoyens et l'Etat. Car si la quintessence de la grécité est censée résider dans ces formes quasi-primitives de l'organisation sociale séculaire préservées, malgré les quatre siècles de "turcocratie" (pouvoir des turcs), et transmises de génération en génération sous le joug ottoman, c'est que celles-ci font appel à des valeurs éternelles de la nation. Mais en revanche, un Etat-Nation moderne ne peut supporter en son sein des formes d'organisation de la violence qui entravent le bon déroulement de l'administration et du respect de la loi souveraine. Ce type de rapports paradoxaux entre la société traditionnelle et l'Etat laisse une grande latitude aux stratégies individuelles dans la vie quotidienne : on peut faire son choix parmi plusieurs registres de valeurs. ${ }^{5}$ L'opinion publique, surtout dans son écho médiatique, nourrie dans ces mêmes méandres relativistes, peut, dans les cas des crimes violents par exemple, basculer soit du côté du bon sauvage, détenteur "naturel" des valeurs de la nation éternelle, soit du côté des lois de l'Etat, expression (civilisée) "culturelle" de la volonté de la Nation.

$$
* * *
$$

Un récent fait divers, tel qu'il a été reporté dans les quotidiens Athéniens, est révélateur ce cette ambiguité de l'opinion vis-à-vis des justiciers crétois.

Le 20 décembre 1988 une affaire de meurtre est jugée en appel dans le tribunal de grande instance de Pirée. Il s'agit de Yannis V., condamné à la prison à vie pour le meurtre de Manolis P. en Août 1983, dans une cafétéria de la ville de Rethymno, en Crète. Le dernier jour du procès, le père de la victime tire sur l'accusé et le tue sur le coup. Les titres des quotidiens, qui ont donné une grande publicité à cette affaire, sont éloquents : "La 'justice' rendue par l'acier brûlant" ; "Le verdict était ... la mort" ; "Vendetta sauvage dans le tribunal". Les reportages sont dans le même esprit : on oscille entre l'admiration pour le vaillant crétois et l'horreur de l'homme urbain face aux mœurs primitives. Dans un journal du soir à grand tirage, on peut lire :

"Impassible et calme, le grand et maigre Crétois nous a parlé, presque ravi, du but qu'il s'était fixé et qu'il a atteint. ...il porte les bottes du costume traditionnel, il a des moustaches toutes noires, il semble sortir d'une page de Kazantzakis, et sa froideur fait glacer le sang ... Sereine et tout-à-fait en accord avec lui, sa femme grande et solide se tient à ses côtés" (Eleftherotypia, 21.12.88).

Dans un autre journal, le meurtrier, juste après son exploit, s'explique :

"Il a tué mon fils, je n'ai pas pu supporter cela et je voulais le trouver pour le tuer. J'ai eu cette chance au tribunal et j'ai pris ma vengeance (ekdhikisi ). Avant de le tuer j'étais malade, maintenant je me sens très bien, puisque j'ai fait mon devoir (chreos ). Le revolver était mon arme pendant la résistance et je le gardais pour défendre mon honneur. Maintenant je n'en ai plus besoin, car j'ai réglé les comptes de mon fils. J'ai fait ma justice (dikio ) pour mon fils et si on m'exécute demain je m'en fous".

Le commentaire du journaliste :

"Ces paroles du vieux-Crétois (Gero-Kritikou ) montrent combien est profondement enracinée l'idée de la vendetta crétoise qui est 
respectée avec dévotion (evlavia) chez les habitants du Magne aussi. Il est clair que .. des mesures s'imposent ainsi qu'un effort de la part des pouvoirs publics pour éliminer ces phénomènes de notre vie sociale" (Apogevmatini, 21.12.88).

Le même journal s'intérroge sur la suite des évènements, en explicant que le premier meurtre correspondait à l'ouverture d'un compte (aniktos logariasmos ) entre les deux familles et que la vengeance du père de la première vicitime ne suffirait peutêtre pas à rembourser (xeplirosi ) celui-ci, la dette étant resté impayée (aplirotos ) pendant cinq ans. Comme dans le cas kabyle (Favret, 1968 : 37) c'est la notion du remboursement de la dette qui est ici associée à la comptabilité de crimes entre deux groupes.

Le justicier de 65 ans a été jugé deux années plus tard dans le même tribunal du Pirée : il a été condamné à 12 ans de prison ferme. Ses parents et amis, visiblement satisfaits, ont accueilli le verdict par des applaudissements (Eleftherotypia, Juillet 1990).

\section{LE VOCABULAIRE DE L'HONNEUR}

Que recouvre cette notion d'honneur à la grecque? Dans l'ethnographie de la Grèce, on a le plus souvent traduit par "honneur" le mot timi, tout en précisant que le terme est polysémique : il désigne, selon le contexte, la valeur d'une personne, le respect dû à une autre, les qualités requises pour la bonne réputation d'un individu ou d'un groupe, la vertu sexuelle d'une femme, enfin, le prix, la valeur d'une chose (Campbell, 1964 : 268). ${ }^{6}$ En effet, le champ sémantique du terme, est centré sur la notion de "prix, valeur" et recouvre deux significations divergentes : "honneur", d'une part et, d'autre part, "prix, ce qu'on paie" (Chantraine, 1984:1120). Ainsi, en grec moderne, on défend son honneur (timi), on paie le prix d'un article (timi) mais on paie le prix de ses actes (timima) et on honore (timo ) Jésus Christ. L'homme honorable est timios (Campbell, 1964:291), celui qui se conforme au code moral de la société est filotimos (litt. celui qui aime la timi ;Cf.Campbell, 1964:295-296, Herzfeld, 1980:343, 1985:289n, Astrinaki, 1986:53).

Dans la société crétoise, le concept qui définit un homme d'honneur, celui qui est respecté par la communauté, est l'eghoïsmos, l'amour-propre. L'homme eghoïstis est capable de défendre son honneur (timi), ses biens (periousia) et, par là même, sa réputation d'homme viril, "qui porte une moustache (moustakalis ) et qui a des testicules (varvatos)".

Un homme dont le comportement est conforme au code de l'honneur est un pallikari, s'il est jeune, ou, s'il est déjà père de famille, un leventis, un homme accompli. Les deux termes renvoient aux valeurs de courage, de la vaillance, de la retenue et de la parole donné. ${ }^{7}$

A l'inverse, le déshonneur (atimia) frappe ceux qui n'ont pas su défendre leur honneur personnel ou celui de leurs femmes ; celles-ci apportent l'atimia dans leur maison lorsque leur conduite sexuelle n'est pas conforme au code de l'honneur. Un homme atimos est celui qui n'a pas d'honneur, qui se soucie peu de sa réputation. Dropi est un terme généralement traduit par "honte" et opposé à eghoismos (amourpropre), dans la mesure où c'est le sentiment qui intervient suite à un échec sur le plan 
de l'honneur; aussi, dropi se réfère en premier lieu au comportement déviant féminin (Campbell, 1964:310).

Dans le cas grec, le langage du pouvoir dominant, celui qui se réfère à l'identité nationale, reprend les termes mêmes du code de l'honneur. La famille, le patrilignage, le territoire du groupe lignager structurent le champ. Au-delà de la famille, chaque individu appartient à la communauté locale et à la nation : ainsi, l'honneur de la communauté ou de la nation est une projection du concept de l'honneur familial (Campbell, 1964 : 317). En effet, Ethnos, la nation, est souvent préféré mais aussi confondu avec genos, la race (non pas dans la dimension biologique du terme, mais dans son sens social) : le patrilignage, ou l'ensemble des patrilignages, descendants d'ancêtres communs. Les homogenis, (litt. issus du même génos) dans le langage administrafif contemporain, désigne les grecs à l'étranger, l'ensemble de ceux qui partagent la même culture : la langue et la religion. La patrie (patridha), aussi, désigne en première instance, le lieu d'origine : dans ce système segmentaire, les patriotes sont d'abord deux personnes nés dans le même village, ou dans la même région. Xénos (l'étranger) s'oppose à dikos (le nôtre) à tous les niveaux de la segmentation : la famille, la communauté villageoise, la nation (Cf. Campbell, 1964:316 ; Handman, 1983:194)

L'honneur associé à la défense du groupe solidaire, de ceux qui partagent les mêmes intérêts et les mêmes valeurs familiales et morales n'est est donc pas en contradiction avec le langage national identitaire, qui assimile le groupe de descendance commune à la nation. Dans les tribunaux crétois, le témoin à décharge qui justifie un meurtre en déclarant que chacun vit pour son honneur (timi) et pour sa patrie (patridha ) n'est pas considéré comme un rigolo. Le langage indigène revendique justement cette filiation : les crimes d'honneur sont-ils autre chose que l'application stricte de valeurs nationales, à savoir la solidarité familiale et la défense de l'honneur, de la renommée du groupe. Traduit dans la langue savante, le langage traditionnel de l'honneur se nobilise. Quelle que soit la façon, soit par l'effort "conscient" du témoin, soit par l'intermédiaire -"inconscient"- du scribe, le résultat est le même : on passe du code communautaire au niveau supérieur, celui du code identitaire, national et homogène.

L'analyse ethnographique de la Crète fournit ainsi un schéma familier pour le spécialiste de la Kabylie, de la Sicile, de la Corse ou de l'Andalousie : un vrai homme est celui qui défend son honneur personnel, celui des femmes qu'il est censé protéger et celui de sa famille. L'homme est responsable de la rénommée de son groupe qui dépend du regard que les autres portent sur lui et les siens. De même, l'honneur est d'abord une façon de se respecter soi-même, une valeur qu'une personne possède à ses propres yeux mais aussi ce qu'elle vaut au regard des autres (Pitt-Rivers, 1983:18). Dans une société de face-à-face, où il y a compétition pour la gloire et le prestige, on existe dans le regard d'autrui : on finit par s'identifier à ce que les autres voient en soi. ${ }^{8}$ Dans ce contexte, un homme qui n'est pas viril n'est pas nécessairement une femme, c'est quelqu'un dont le comportement n'est pas conforme à son statut social. C'est la conformité aux normes de la société qui compte avant tout pour définir la renommée, le prestige de quelqu'un. Ne pas se conformer au code moral est une déviance par rapport à la normalité masculine : on dit d'un tel homme qu'il est sans honneur (atimos ), qu'il est lâche (dilos) ou bien qu'il est cocu (keratas ). 


\section{LES CRIMES D'HONNEUR : DEUX EXEMPLES CRÉTOIS}

"Je ne savais pas que ma soeur était enceinte car si je le savais j'aurais tué Vassilis (son amant) et ma soeur ne serait pas dans la tombe à l'heure qu'il est; en tout cas Vassilis, je vais lui casser la gueule. Si mon père ne l'avait pas égorgée (esfaze ) je l'aurais fait moi-même. Si jamais j'entendais dire que mon père avait touché ma soeur je lui crèverais les yeux. Après le meurtre que mon père a fait j'ai senti une sérénité (galini), comme la ${ }_{9}$ même chose est arrivée à mon père" (Témoin à décharge, Cas no 7, 1971).

"Je suis gendarme de métier. Selon la mentalité de ces gens-là, la jalousie suffit pour qu'ils tuent quelqu'un. Selon les dires des gens, aucun des crimes (egklimata) commis n'a eu lieu pour une raison sérieuse (dhia sovaron logon ) " (Témoin à charge, Cas no 51, 1982).

Les deux cas présentés ici sont tirés des archives des comptes-rendus d'audience. On peut dire que ce sont des cas-limites. Le premier, c'est le crime d'honneur-type, parfaitement licite : la femme qui déshonore la famille est éliminée. Le second est en apparence un exemple de crime gratuit : un homme tue un membre de la famille rivale sans raison valable. Dans le premier cas, les témoins sont tous à décharge; dans le second, c'est contre l'accusé que se fait l'unanimité, à tel point que lui-même se présente au tribunal répenti et honteux.

\section{la fille égorgée}

"Les étalons (begiria ${ }^{10}$ ) sont libres, comme on dit ici en Crète, ce sont les juments (foradhes ) qu'il faut attacher" (témoin à décharge).

Le premier cas (no 7 du corpus) de la fille égorgée par son père s'inscrit dans une catégorie bien connue en matière de crime d'honneur méditerranéen. Tout homme d'honneur est tenu d'égorger la fille qui déshonore le nom de la famille (Favret, 1968, Campbell, 1964). Ici, les faits prennent place dans une situation tout-à-fait "ordinaire" d'exode rural -- qui tourne mal. Le père décide d'envoyer sa femme en ville pour que ses deux enfants puissent apprendre un métier ; la fille est placée comme apprentie chez une couturière, et le mari de celle-ci la séduit. Les rapports adultères du couple durent quelques années et sont connus par les deux familles : ainsi, le frère témoigne avoir surpris sa sœur avec son amant et de l'avoir chassé avec des pierres, et la couturière elle-même envoie la mère et la fille chez le gynécologue pour qu'il répare les "dégats". ${ }^{11}$ Seul le père est censé ne rien savoir : on lui cachait les faits "pour qu'il n'y ait pas de meurtres" (fonikà ). Quoi qu'il en soit, la famille décide le marier la jeune fille déshonorée avec un homme pauvre : "Pour couvrir ses méfaits (pompès, litt. actes qui entraînent le ridicule devant l'opinion publique), on a trouvé son fiancé Giannakis et on l'a fiancée et on lui a promis un terrain en ville (oikopedho ), des meubles et des habits et on lui a tout fait." Mais pendant les fiançailles, la fille a continué ses rencontres clandestines avec son amant et le fiancé a tenté de profiter de la situation : sachant que la fille était enceinte, il demanda plus de dot (panorpiki, la sur-dot, réservée à contrebalancer un défaut de la fiancée) et, lorsque la famille ne cèda pas, il rompa les fiançailles. La fille est enceinte de cinq mois, sans mari en vue et c'est alors que le père entre en scène : quasiment absent pendant le déroulement des négotiations avec le futur 
gendre, il prend cette fois-ci les choses en main et tue sa fille avec un couteau de cuisine.

Le père intervient pour défendre son honneur "J'ai tué ma fille pour des raisons d'honneur (logous timis) ", car il est vrai qu'en Crète, comme en Espagne, "l'offense extrême à l'honneur d'un homme ne se réfère pas à sa propre conduite, mais à celle de sa mère, sa sœur ou sa fille, qui sont censées avoir le même sang" (Pitt-Rivers, 1991:28). Les témoins qui viennent déposer tiennent le même discours :

Le père d'une telle fille dans notre village n'a pas de repos, et on exécute la fille et son ami. L'accusé était un homme honorable (timios) et paisible (filisichos), il n'a jamais mis les pieds dans une salle de tribunal.

Les termes employés dans ce contexte se réfèrent à l'opposition honneur/déshonneur selon qu'il s'agit de l'homme ou de la femme. Ainsi, le père est honorable (timios ) pour avoir défendu l'honneur de sa maison, mis en danger par le comportement de sa fille, sans vergogne (dropi). Car dropi est bien le terme qui convient à la fois au "sentiment de vergogne qui garantit l'honneur de la femme" (PittRivers, ibid.) et au résultat de cet état des choses : le déshonneur de la maison qui rejaillit sur le chef de famille. ${ }^{12}$

Avec ce déshonneur (dropi ) qu'elle m'a fait je ne pouvais pas vivre dans mon village. ... Toute ma vie j'ai été un monsieur (kirios ) et un très bon père de famille (ikogeniarchis ). ... La Bible (evaggelio ) dit 'tu ne tueras point' mais si on marche avec la Bible, c'est le diable qui va nous emporter (mas pire o diaolos ).

La honte infligée à une maison par la conduite de la femme est asocié à une pollution : on "lave" (xepleno), on purifie du déshonneur en égorgeant (sfazo) la fille tout comme on invoque la grâce des saints et leur médiation pour faire pardonner ses péchers en sacrifiant les animaux (sfaghia). :

... A mon avis en l'égorgeant (sfaxi) il a lavé le déshonneur de sa maison (xeplithike i dropi tou spitiou tou).

Le terme sfazo est en effet évocateur de l'état de souillure et de purification rituelle qui semble être le contexte de ces affronts à l'honneur masculin. C'est le mot qui exprime, en premier lieu, l'idée d'égorger dans le contexte du sacrifice, et qui peut également signifier "abattre", "massacrer" (Chantraine, op.cit., 1073).

Pitt-Rivers évoque "l'état de pollution où l'on entre par le fait de se laisser priver de son privilège et de se dérober à son devoir" lorsqu'on devient cocu et, dans ce contexte, "la moquerie qui est le sort du cocu ... ne cherche pas à punir une infraction, mais à signaler un état rituel de pollution" (1991:30). Justement, un des témoins crétois situe l'affaire du meurtre de la fille par son père dans ces mêmes termes :

"Aucune (femme) qui a fait de telles choses (tetia pramata) dans notre région n'a survécu, celui qui ne la tue pas on l'appelle cocu (kerata )".

Les témoignages recueillis pendant l'audience au tribunal de grande instance sont presque tous concordants : le père a tué sa fille car il n'avait pas d'autre issue. 


\section{l'homme exécuté}

Le deuxième cas s'inscrit dans le cadre de l'échange de violences entre deux familles rivales. Le meurtre jugé durant l'audience en question est d'emblée considéré comme un acte non conforme aux normes de la compétition pour l'honneur.

Un premier épisode, six années auparavent, est peut-être à l'origine du meurtre : lors d'une bagarre, un membre de la famille Canaki a frappé un membre de la famille Garidaki avec une chaise. Ce dernier est mort quelques mois plus tard d'un cancer au cerveau. Les parents de la première victime ne donnent alors aucun signe extérieur de vouloir se venger et les choses en restent là. Selon les témoins (à charge et à décharge), il n'y avait aucun "différend familial" ; l'incident de la chaise n'avait pas changé les relations entre les deux familles. Mais apparemment, la famille Garidaki attendait son heure pour frapper : un mois avant le meurtre, le comportement de ses membres change à l'égard de la famille Canaki : "L'accusé a changé son comportement envers nous. Il nous cherchait des histoires" (témoin à charge, frère de la victime). Pour les parents de la victime, c'est toute la lignée des Garidaki qui aurait décidé en commun ce meurtre contre les Canaki :

"A mon avis les Garidaki étaient jaloux (zilevan) de la victime parce qu'il était conseiller à la préfecture, qu'il avait une grande fortune (periousia) et ils ne voulaient pas qu'il soit entre leurs pattes. Les autres années ils n'avaient personne pour faire ce boulot. ... La victime était le plus illustre (epifanis) de la famille Canaki. Ils ont choisi le pire (chirotero )pour tuer le meilleur (kalitero )" (témoin à charge, cousin de la victime).

La situation est analogue aux cycles de meurtres qui prennent place entre deux lignées ambitieuses en concurrence pour le pouvoir en Kabylie. Le "pire" de la lignée des Garidaki ressemble beaucoup au "voyou irresponsable qui lance les cycles", dont doit disposer une lignée qui cherche à s'affronter (Favret, 1968).

Les normes du code traditionnel sont le vrai enjeu dans le cas examiné ici : tout indique, en effet, que le comportement de la victime est conforme aux normes et que l'accusé arrive à ses fins en les détournant. La première règle baffouée est celle de l'égalité en honneur : ce n'est que les égaux qui s'affrontent et qui peuvent lancer et relever les défis. Or, l'accusé est manifestement pas l'égal de la victime et celle-ci ne le prend pas au sérieux. L'accusé lui-même admet cet état des choses : "c'est par respect que j'ai été le saluer". Pour les témoins, la victime était un homme riche et respecté, tandis que l'accusé "était un homme quelconque (sinithismenos ) du village". Selon le cousin de la victime, interrogé sur les coups échangés, celle-ci n'aurait pas daigné (katadechotan ) de le frapper. Il refuse, ainsi, dans un premier temps, de relever le défi (l'accusation du vol); car un défi qui reste sans réponse devient déshonorant pour celui qui le lance, puis il traîte son agresseur comme un inférieur ("va te faire foutre (asiktir ), ${ }^{13}$ disparais de ma vue"). C'est ce que cherche l'assusé, car il lui faut une excuse valable pour réagir de manière violente : "quand j'ai entendu cette insulte (vrisià ) j'ai commencé à perdre la raison (na ta chano )". Jusqu'à la fin, les provocations de l'accusé sont reçues avec mépris : quand il lance "si tu es un homme (andras), attends que je revienne", la victime ne répond pas. "Mon oncle soit ne croyait pas qu'il reviendrait, soit il ne croyait pas qu'il le tuerait. Lorsque les clients du café lui ont dit de partir, il a répondu que nous (c'est-à-dire les hommes de la lignée Canaki) n'avons pas l'habitude de nous cacher". 
Le jeu de la parole donnée est ici perfide : dans une société où l'apparence est aussi -sinon plus- importante que la réalité, le calcul du meurtrier et de la future victime se porte sur le dire et le faire : reculer dans une telle situation, pour l'agresseur comme pour l'agressé, équivaut à une perte permanente de l'honneur. La victime était coincée : elle n'a pas bougée, et le meurtrier est revenu et lui a tiré cinq balles en pleine figure.

\section{USAGES DU LANGAGE : QUELS MOTS POUR LE DIRE?}

Le langage de l'honneur, lorsqu'il franchit la porte des tribunaux crétois, emprunte la koinè savante pour devenir légitime. Les termes vernaculaires cèdent leur place aux mots de la langue savante qui renvoient au registre moral national. ${ }^{14}$ Ici comme ailleurs, la langue officielle imposée à tous les ressortissants de l'unité territoriale comme la seule légitime, devient le moyen de communication entre les citoyens, témoins, acteurs, juges et jurés, dans l'espace officiel du tribunal (Bourdieu, 1982:27). Le langage scolaire est un véhicule privilégié : il est employé surtout par les habitants de la ville, qui ont atteint un niveau d'éducation élevé. Pour prendre la formulation de Bourdieu :

"Le maître d'école est maître à parler et maître à penser (...) En apprenant aux enfants la même langue, une, claire et fixée, il les incline déjà tout naturellement à voir et à sentir les choses de la même façon et il travaille à édifier la conscience commune de la nation. (...) Le code, au sens du chiffre, qui régit la langue écrite, identifiée à la langue correcte, par opposition à la langue parlée, (...) acquiert force de loi dans et par le système d'enseignement" (1982:32-33)

Ainsi, un gendarme parle de son cousin assassiné comme le membre le plus "illustre" (epifanis ) de sa famille, la couturière dont l'apprentie couche avec son mari explique au jurés qu'elle a tenté de "raisonner" (noutheto ) la fille "animée" (zoÏri ) et "frivole" (epipoléa).

Une analyse de contenu de quelques comptes-rendus d'audience ${ }^{15}$ donne des résultats intéressants. On constate en effet une vraie pénurie de termes vernaculaires relatifs à l'honneur, comme si les acteurs, en s'expliquant devant le tribunal, censuraient, non seulement les mots, mais aussi le code traditionnel de l'honneur devant cette assemblée officielle.

Tout d'abord, le mot timi (honneur) et ses dérivés se font rares. C'est seulement dans le cas de la fille égorgée par le père qu'on évoque timi deux fois. En revanche,atimia (déshonneur) et atimazo (déshonorer) reviennent un peu plus souvent : 5 locutions en tout.Timios (honorable), enfin, n'apparaît qu'une seule fois, pour désigner le même père assassin de sa fille.

Timi , l'honneur au sens général, appartient plutôt au registre savant, et par là même, au langage du pouvoir. Ce n'est que dans le cas de l'honneur sexuel, lorsque l'honneur d'une femme est en jeu, que l'on utilise le terme timi : ainsi, le père qui tue sa fille enceinte des œuvres de son amant évoque sa timi, son honneur en tant qu'homme protecteur de la famille. Par contre, l'utilisation du terme atimia (déshonneur) ou atimos (adjectif, sans honneur) est plus souple et on le voit apparaître dans deux contextes différents. D'une part, dans le cas de la femme qui inflige une atimia à sa famille par son comportement. D'autre part, le terme est utilisé pour indiquer qu'un homme n'a pas d'honneur, pas de parole : dans le cas no 51, par exemple, l'auteur du 
crime accuse un des témoins de mentir: "il est menteur et sans honneur" (ke atimos ine ke pseftis ine ).

Sorti de la timi, les concepts de base du code de l'honneur dans le contexte crétois ne sont pas évoqués dans les salles des tribunaux. L' eghoïsmos, l'amour-propre masculin (terme-clé pour l'analyse du comportement masculin dans la monographie de Herzfeld) n'apparaît pas, tout comme la parexighisi, le "malentendu", conséquence d'un conflit ancien et déclencheur potentiel de violences (Astrinaki, $1986: 142$ ). Le mot andras (homme) est employé dans le sens d'"époux" de manière courante ; le sens d'homme viril reste exceptionnel. Même andrismos (virilité), terme pourtant usuel dans la koinè ne fait que des rares apparitions dans le corpus judiciaire. Enfin, les autres termes relatifs aux vertus viriles répertoriés dans l'ethnographie crétoise, sont également absents : pallikari, le gaillard, l'idéal du jeune homme n'est pas plus mentionné que leventis, le vaillant, l'homme viril accompli.

De cet ensemble, seuls deux termes font leur apparition, et cela dans un sens négatif : dans le cas du meurtre dans le café, un des témoins déclare avoir dit à l'accusé "Fils de pute, tu veux jouer au petit mec' (poutanas yé, tora tha kamis to andraki ). Le terme employé est non pas andras (homme) mais andraki (litt. petit homme), qui désigne le "moins qu'homme". Dans le même cas, un autre témoin décrit l'accusé dans ces termes : "On l'a désigné comme le premier gaillard (protopallikaro ) de sa lignée et c'est pour cela qu'on l'a choisi pour tuer". Dans le contexte de la déposition de ce témoin à charge, l'usage du mot "protopalikaro" est ironique. En effet, l'accusé aurait peut-être bien voulu être considéré comme le gaillard, le justicier de sa lignée, mais en fait il a perdu sur les deux plans : il s'est fait arrêté et jugé et, en plus, son lignage ne s'est pas montré solidaire.

Il en va de même pour le terme soï (vocable turc qui désigne la lignée agnatique, utilisé couramment dans la langue vulgaire dans le même sens), qui se trouve remplacé par ikogenia , mot qui désigne la famille tant dans la langue "pure" qu'en démotique. Pourtant, le plus souvent, c'est le patrilignage qui est évoqué ici par ce terme général : "C'est Nicos, Kostas et Lefteris Garidakis ( ses oncles et cousins patrilatéraux au troisième degré) qui ont désigné l'accusé pour tuer mon frère ... l'accusé était considéré comme le plus vaillant (protopalikaro ) de la famille (ikogenia) et c'est pour cela qu'ils l'ont choisi" (témoin à charge dans le cas no 2). Dans le même cas, la victime aurait insulté l'accusé par les mots "Je baise ta 'famille', bâtard" : ici encore, on "traduit" le vocable étranger "soÏ" par "ikogenia ". En effet, l'insulte suprême -et courante- est bel et bien "je baise ta lignée (sö̈ )", c'est-à-dire toi et l'ensemble de tes agnats.

Dans le cadre de la compétition masculine, ce sont en effet deux lignées qui s'affrontent à travers leur membres : c'est l'honneur de toute la lignée qui est en jeu, chacun agissant au nom de son groupe. Ainsi, dans le cas du meurtre dans le café, la réponse que la victime aurait donnée au défi lancé par l'acusé "Si tu es un homme attends que je revienne" c'était "les hommes ne partent pas, nous n'avons pas appris à nous cacher". Nous, c'est la lignée des Canakis, les agnats qui portent le même nom. Dans son témoignage, le fils du frère de la victime, relate les faits en se considérant manifestement comme faisant partie du nous : "mon oncle a répondu que nous n'avons pas l'habitude de nous cacher".

Or, il arrive que la lignée ne se montre pas solidaire des actes de ses membres. Dans le cas examiné ici, c'est ce qui arrive à l'accusé. Aucun membre de son patrilignage ne vient témoigner, à l'exception de son père. Il est évident que les 
Garidaki ne veulent pas assumer leur responsabilité collective, ne veulent surtout pas cautionner l'ouverture d'un "compte" entre les deux lignées ; mais pour la partie civile c'est l'ensemble des Garidaki qui sont les auteurs moraux du crime :

"Depuis que j'était enfant j'entendais dire que la famille des Garidaki était mauvaise ( $k a k i$ ). Ceux-ci (les Garidaki, note du scribe) ont toujours empiété sur notre propriété (periousia). Nous, on avait 1500 brebis et ils étaient eux aussi des bergers et ils voulaient nous chasser de nos terres " (témoin à charge, épouse de la victime).

"Eux (la famille de l'accusé) ont cru qu'ils allaient prendre notre patrimoine (periousia) là-bas et spécialement l'oncle de l'accusé Nicolas Garidakis. .. C'est Nicolas, Kostas et Lefteris Garidakis qui ont poussé (evalan ) l'accusé à tuer mon frère, tout le monde le disait .. Eux ils offraient de l'argent mais ils ne trouvaient personne pour tuer mon frère, ils le guettaient et ils ne faisaient rien et c'est alors qu'ils ont décidé de le tuer eux-mêmes de manière provoquante ... Je dis maintenant que l'accusé est sans expérience (protopiros ) et sans importance (asimantos ). Il n'avait aucune raison de tuer mon frère tout seul. On l'a trouvé en soldes (se timi efkerias ) pour tuer le lion (liontari ) mon frère, cette espèce de déchet (skivalo )"(témoignage du frère de la victime).

Même les insultes, qui apparaîssent, on l'a vu, entre guillemets, et qui apportent la preuve de la provocation, du défi dans le jeu de l'honneur, sont réduites au strict minimum. Ici ce n'est pas le scribe qui intervient, mais les acteurs eux-mêmes; on n'ose pas prononcer ces paroles devant les autorités policières et judiciaires, par "honte" (dropi ) ou par "peur" (fovos ) des conséquences. Ainsi, tel accusé déclare (cas no 54):

"ce jour-là la victime m'a approché et m'a dit 'ici sale pédé je vais te dire, je t'arracherais la moustache poil par poil et je vais te faire le travail (c'està-dire 't'enculer', NDT)". Ce dernier j'ai eu honte (drapika) de le déposer à la police".

Et un témoin :

"Pendant la bagarre, $\mathrm{C}$ a dit à $\mathrm{G}$ 'je baise ta famille, batard' ... je n'ai pas dit cette phrase au début parce que j'avais peur (fovithika)".

C'est plus particulièrement les expressions à connotation sexuelle qu'on évite scrupuleusement dans les salles des tribunaux. Ici c'est l'usage de la katharevousa, avec son arrière-goût scientifique et médical qui s'impose. Le romancier Taktsis décrit une scène qui se déroule au tribunal d'Athènes des années 1930; le juge demande des précisions sur une affaire d'adultère : "dis-nous seulement jusqu'où ils étaient arrivés. En katharevoussa ". Puis: "dis-nous maintenant ce qu'il c'est passé après - en dimotiki " (Le troisième anneau, 1974:169) .

La censure et la traduction de termes "vulgaires" en mots de la katharevousa est aussi un moyen de rendre licites et intelligibles des conflits qui appartiennent à un code de comportement traditionnel. En 'traduisant' la parole des acteurs dans la langue savante, on se situe d'emblée dans le système de valeurs qui est propre à ce discours, 
celui de la société urbaine et moderne. Ainsi, les faits rapportés devant le tribunal le sont souvent "hors contexte".

Or, le décalage entre langue et langage n'est pas toujours évident. Car, en effet, si les crimes commis pour l'honneur d'une femme paraîssent moins "étranges", c'est que les catégories du licite et de l'illicite sont, sinon communes, au moins intelligibles dans les deux langages. Un bon père de famille n'accepte pas que sa fille devienne une traînée ; une femme adultère doit être punie. Le code moral de la société réprouve ce comportement et la loi permet de punir la déviance; même si elle n'autorise pas l'individu de faire justice lui-même. Par contre, la compétition masculine pour l'honneur qui sous-tend les cycles de violences entre lignées se structure autour d'un code de valeurs qui ne sont pas celles de la loi ni de la morale nationale. Dans ces cas, étrangers au langage de la justice, le rôle des jurés est celui de médiateurs : citoyens nourris dans le respect de la tradition, souvent originaires des communautés villageoises, ils représentent, aux yeux des acteurs, l"opinion publique".

"L'opinion publique dit clairement que le plan de l'assassinat a été établi après une décision commune de Nicos, Kostas et Lefteris Garidaki." (cas $51)$.

"L'opinion publique dit que la victime a traité l'accusé de pédé" (Cas 54).

Au coeur de l'opposition entre les deux langages, les verdicts rendus par les tribunaux sont-ils l'expression de l'opinion publique, qu'évoquent les intéressés? Ces décisions sont en effet conformes à une "opinion publique" qui n'accepte pas le code traditionnel de l'honneur. Lorsqu'on tue pour tuer, parce qu'on a des "différends familiaux" on est puni comme n'importe quel criminel. Par contre, lorsqu'on élimine celle -ou celui- qui déshonore la famille par des actes non conformes à la morale on est jugé avec clémence. Le père ou mari qui tue sa fille ou sa femme qui le déshonore est sûr de sortir de prison au plus tard au bout de dix ans, tandis que le berger qui tue son voisin risque la prison à vie.

Les jurés tirés au sort sont, en quelque sorte, les représentants d'une communauté intermédiaire, villageoise et citadine à la fois. Dans la Crète d'aujourd'hui, d'urbanisation récente et rapide, on peut difficilement parler de communauté villageoise isolée, solidaire et distincte. Les familles qui s'installent en ville laissent des parents et des terres au village; les familles villageoises ont toujours des proches parents en ville. L'opinion publique évoquée dans les tribunaux se forge donc dans un espace nouveau, où les valeurs citadines et celles de la société communautaire se côtoient. Dans ce contexte, il n'est pas étonnant de constater que le lieu de la compétition masculine n'est plus l'espace villageois, que la compétition se passe ailleurs, dans les centres urbains devenus accessibles à tous.

Tout se passe donc comme si les témoins qui viennent à la barre du tribunal refusaient de mettre ce qui fait l'identité masculine dans le marché linguistique : le tribunal relève du monde insitutionnel, des instances du pouvoir. C'est le lieu des "eux", pas des "nous". Si on ne nomme pas les vertus du paradigme masculin en l'objectivant, on parle volontiers de l'honneur perdu de la femme, en parlant d'elle à la troisième personne. Car s'il est vrai que l'honneur de l'homme passe à travers la conduite sexuelle 
de ses femmes dépendantes (fille, sœur, ou épouse), il passe justement à travers une tierce personne. L' intégrité morale et physique de l'homme n'est pas atteinte lorsque la femme qu'il protège est fautive, sauf si il ne réagit pas. C'est alors seulement que son honneur personnel sera mis en jeu.

Il en va tout autrement dans les cas des défis et contre-défis qui s'inscrivent dans le cycle des violences, hors contexte sexuel, entre individus ou entre lignages, dans la compétition masculine "pure". Ici, il s'agit de l'honneur personnel de chacun et il faut employer le "je" : même répéter au tribunal une insulte lancée contre soi, c'est comme si on l'endossait sans parvenir à punir l'agresseur. C'est pourquoi les insultes sont non pas évoquées directement, mais toujours rapportées par des témoins. Car au tribunal, on refuse de parler des choses qui appartiennent au "nous", au code du comportement masculin, à l'homme individuel dans son intégrité, qui n'ont cours qu'à l'interieur de la communauté. Et lorsqu'on est obligé de le faire, on évoque les faits dans la langue des autres, en katharevoussa .

Consciente de sa différence, la communauté refuse de se comparer à la morale nationale pour ce qui touche le plus profond d'elle-même : l'identité masculine.

\section{Maria COUROUCLI - CNRS}

Laboratoire d'Ethnologie et de Sociologie Comparative

Université de Paris X-Nanterre 


\section{ARTICLES ET OUVRAGES CITES}

ASTRINAKI, U. Honneur et violence dans la Crète contemporaine, Mémoire de maîtrise, Université de Paris X-Nanterre, 1986.

BABINIOTIS, G "A linguistic approach to the 'Language Question' in Greece", Byzantine and Modern Greek Studies, 5, 1979.

BLACK-MICHAUD, J. Cohesive force, Feud in the Mediterranean and the Middle East, Oxford, Blackwell, 1975.

BLOK, Anton "Rams and billy-goats : a key to the mediterranean code of honour", Man, 16, 3, 1981.

BOURDIEU, P. Ce que parler veut dire, Paris, Fayard, 1982.

BOURDIEU, P. Le sens pratique, Paris, Minuit, 1980.

CAMPBELL, J.K., Honour, family and Patronage, Oxford, Clarendon, 1964.

CHANTRAINE, P. , Dictionnaire étymologique de la langue grecque, Paris, Klincksieck, 1984.

FAVRET, J. "Relations de dépendance et manipulation de la violence en kabylie", L'Homme, 8, IV, 1968.

FERGUSON, C.A. "Diglossia", Word, 15, 1, 1959.

HERZFELD, M. Anthropology through the looking-glass : Critical ethnography in the margins of Europe, Cambridge, C.U.P., (1987), 1989.

HERZFELD, M. The poetics of manhood : contest and identity in a cretan mountain village, Princeton, Princeton University Press, 1985.

KAZAZIS, Kostas, "Learnedisms in Costas Taktsis's Third Wedding", Byzantine and Modern Greek Studies, 5, 1979.

KAZAZIS, Kostas, "The status of turkisms in the present-day balkan languages", in Birnbaum,H. \& Vryonis, S. (eds) Aspects of the Balkans, The hague, Paris, Mouton, 1972.

LABOV, W. Sociolinguistique, Paris, Minuit, 1976.

LE ROI LADURIE, E. Montaillou, village occitan de 1294 à 1324, Paris, Gallimard, 1975.

MIRAMBEL, A. Petit dictionnaire Français-Grec moderne et Grec-Moderne-Français, précédé de notions de lecture et d'écriture du grec moderne et d'un résumé grammatical. Nouvelle édition, Paris, Maisonneuve et Larose, 1980.

PATLAGEAN, E. "Discours écrit, discours parlé. Niveaux de culture à Byzance au VIIIe-XIe siècles", Annales, ESC, 2, 1979. 
PERISTIANY, J. (ed), Honour and Shame, London, Weidenfeld and Nicolson, 1965.

PITT-RIVERS, J.Anthropologie de l'honneur, La mésaventure de Sichem, Paris, Le Sycomore, 1983.

PITT-RIVERS, J. "La maladie de l'honneur", in M. Gautheron, L'honneur, Paris, Editions Autrement, 1991.

TAKTSIS, K. To Trito Stefani (Le troisième anneau), Athènes, Ermis, 1974.

TSOUDEROS, Y., Chants funèbres crétois, Athènes, 1976 (en grec).

VASSILIKOS, Vassili, "Quand la langue est le message", Autrement-Grèce, Série Monde, no 39, 1989.

VERNANT, J-P. "La 'belle mort' d'Achille", in M. Gautheron, L'honneur, Paris, Editions Autrement, 1991. 


\footnotetext{
${ }^{1}$ Sur le phénomène de la 'diglossia' voir l'article comparatif de Ferguson (1959)

${ }^{2}$ Les cas cités ici sont empruntés d'un corpus d'archives judiciaires de Crète qui ont fourni la base d'un travail de maîtrise soutenue par U. Astrinaki à l'Université de Paris X-Nanterre en 1986.

${ }^{3}$ E. Le Roy Ladurie, 1975.

${ }^{4}$ Sur la relation entre le code de l'honneur et l'Etat, voir A. Blok (1981:434) qui explique l'importance de la force physique dans le discours de l'honneur en méditerranée par l'absence de centres politiques stables capables de protéger la vie et les biens des gens.

${ }^{5}$ Cette situation d'ambiguité qui caractérise la manière de 'dire' et de 'faire' et, faut-il ajouter, d'être et de paraitre a été décrite sous le concept quelque peu réducteur de 'disemia' par Herzfeld (1987).

6 Pour les diverses significations du terme latin, voir Pitt-Rivers, 1983:17-35)

${ }^{7}$ Voir notamment Campbell, 1964: 168-272, Astrinaki, 1986: 45-46.

${ }^{8}$ Voir J.P. Vernant, 1991.

${ }^{9}$ Fond 'Dossiers judiciaires crétois', Labethno-Nanterre DJC, 7 et 51. Les noms qui apparaîssent dans tous les textes cités ici ont été changés, ainsi que les dates exactes.

${ }^{10}$ Beygir, en turc : les chevaux non castrés

${ }^{11}$ Il est courant que les filles déflorés aient recours à une parthenorafi (litt. couture de vierge), c'est-à-dire à une intervention médicale qui vise à recoudre l'hymen rompu, le plus souvent juste avant le mariage ou les fiançailles, pour que le futur époux ne se rende pas compte de l'état de sa promise. On dit alors qu'une fille a été "cousue" (tin erapsan).

${ }^{12}$ Dropi est à la fois la honte et le sentiment de vergogne. Ainsi, adiantropi (sans vergogne) est la femme dont le comportement est sexuellement provoquant ; on peut lui lancer alors, "tu n'as pas de dropi" (honte), le sentiment de vergogne. Un homme ou une femme aura 'honte' (dropi) de ses actes non conformes, puisqu'ils entrainent une perte de prestige (Voir aussi Campbell, 1964:310 et Herzfeld, 1985:233 qui oppose dropi à eghoismos, l'amour-propre masculin).

${ }_{13}^{13}$ Expression turque ("Ha siktir" = "va te faire baiser", sik = "bite") utilisé en grec comme une insulte "moins forte" que l'expression grecque équivalente "gamoto" (Sur l'emploi des termes turcs dans la langue grecque et notamment des insultes et leurs 'poids' variable, voir Kazazis,1972).

${ }^{14}$ Sur l'usage du vernaculaire au sein d'une communauté restreinte, ayant ses propres normes linguistiques et sociales, voir l'analyse de Labov (1976) à propos du parlé noir-américain

${ }^{15}$ Dans ce corpus qui comporte 97 cas au total, sept ont été choisis parmi les plus complets du point de vue documentaire pour pratiquer cette analyse. Deux sont des cas de crimes pour l'honneur d'une femme, deux autres crimes sont à classer dans la catégorie de cycles de violences entre lignages, les trois derniers étant des crimes plus 'banales'.
} 